\title{
The Potential Role of Antigen Priming In Increasing the Overall Antimicrobial Resistance in Captive Silver Foxes
}

\author{
Spînu Marina*, Niculae Mihaela, Pall Emoke, Brudaşcă Florinel, Vasiu Aurel, Cerbu Constantin, \\ Giupana Radu, Șandru Carmen Dana
}

Department of Clinical Sciences - Infectious diseases, University of Agricultural Sciences and Veterinary Medicine, Str. Manastur no.3-5, Cluj-Napoca, ROMANIA

\begin{abstract}
Objective: We hypothesized antigenic priming should stimulate the humoral and cellmediated adaptive immunity, further improved by vegetal extracts, alleviating the immune suppression induced by captivity stress in silver foxes. Material and Methods: One of two groups of adult silver foxes was sc primed and boosted 7 days later with a $5 \%$ SRBC suspension. Serum antibody titers (hemagglutination test) and circulating immune complexes levels (4.2\% PEG precipitation) were quantified and In of the antibody titers were calculated seven days later. An in vitro blast transformation test was carried out on blood samples using alcoholic extracts of $C$. officinalis, $A$. montana, S.officinale, Echinaceea spp. and immune stimulating compounds and glucose consumption was evaluated. The significance of the differences was interpreted by Student's t-test. Results: Cells grew better in primed foxes versus the unprimed individuals $(68.8 \pm 9.88$ and $17.49 \pm 22.9)$. The primed animals reacted significantly $(p<0.001-p<0.01)$ better to vegetal extracts (C. officinalis, $E$. angustifolia) and to thymus extract, selenium salts and bovine tuberculin. Anti-SRBC antibodies were highly variable (0.69-5.54). Conclusion: SRBC exerted a positive effect on both humoral and cell-mediated immune responses in silver foxes, supporting the enhancement of antimicrobial defense by booster vaccination and immune stimulating therapy.
\end{abstract}

Keywords: Silver Foxes, Antigenic Stimulation, Adaptive Immunity, Vegetal Extracts.

\section{INTRODUCTION}

The immune system links the individual to its microbial environment and the outcome of this interaction is vital for the survival. Environmental factors, including farming technology, act as stressors on the immune system, and the timeframe and degree of adaptation is highly dependant on the intensity and duration of the stressors' action. The impaired immune response in individuals subjected to various stress factors can cause: low protective responses following vaccination or concurrent infections/diseases due to lower protection, as well as economic losses. Modern immunology is seeking for alternative immune stimulating compounds for which plants could represent a source.
Scientific data support the healing activity of plants indicating that, unlike conventional drugs, which sometimes aleviate the symptoms without removing the cause, natural remedies eliminate the cause. ${ }^{1-8-13,14}$ Stimulating effects of plant extracts were scarcely studied in wild carnivores. At our best knowledge, there are no experiments carried out in Silver foxes aiming to prove the combined effects of antigen priming and medicinal plants on their immune system. We hypothesized that antigenic priming should stimulate the humoral and cell-mediated adaptive immune response, being further improved by vegetal extracts,
DOI: 10.5530/ijper.51.3s.37 Correspondence: Spînu Marina, Department of Clinical Sciences - Infectious diseases, University of Agricultural Sciences and Veterinary Medicine, Str. Manastur no.3-5, Cluj-Napoca, ROMANIA

Contact: +40 $264596384 / 173$ E-mail: marina.spinu@gmail. com

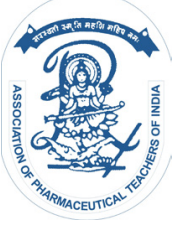

www.ijper.org 
diminishing the degree of immune suppression induced by captivity stress in silver foxes.

\section{MATERIALS AND METHODS}

The research was carried out on two groups of adult, antigen primed $(\mathrm{n}=16)$ and unprimed $(\mathrm{n}=14)$ silver foxes. The priming was performed by s.c. injection (day 0 ) and booster (day 7) with $0.5 \mathrm{ml}$ a 5\% SRBC suspension. On day 14, blood was sampled for serum and on heparin $(50 \mathrm{IU} / \mathrm{ml})$ from all animals.

The anti-SRBC antibody titers were assessed by a hem agglutination test according to the OIE ${ }^{16}$ protocol, in 96 " $U$ " shaped well plates, performing two fold dilution of the serum $(1 / 2,1 / 4, \ldots)$ in saline and adding $0.025 \mathrm{ml}$ of a $5 \%$ SRBC suspension/well. After $2 \mathrm{~h}$ of incubation at $37^{\circ} \mathrm{C}$, the last dilutions with positive results were calculated as $\ln$ of the titers.

To establish the circulating immune complexes levels (CIC) a $4.2 \%$ PEG precipitation test was applied, mixing $196.7 \mu \mathrm{l}$ of PEG and $3.3 \mu \mathrm{l}$ serum in 96 flat bottom plates, and followed by spectrophotometric reading at $450 \mathrm{~nm}$, $\mathrm{d}=0.5 \mathrm{~cm}$ after $60 \mathrm{~min}$ of incubation. The CIC values in units $(\mathrm{U})$ were calculated according to the formula: $(\mathrm{U})$ $\mathrm{CIC}=$ (sample E- control E) $\times 1000$.

Lenkocyte blast transformation test. ${ }^{6}$ The leukocyte blast transformation test measures the in vitro reactivity of mononuclear cells to sensitizing antigens. Commercial alcoholic extracts for human use of Calendula officinalis, Arnica montana, Symphytum officinale, Echinaceea purpureea, Echinaceea angustifolia (Plantextract, Romania) produced according to the German Homeophatic Pharmacopeia, were used to treat the cultures. Similarly, an aqeous thymus extract, commercial Seleretard, balsam of Peru and bovine tuberculin, as immunstimulators.
One ml of each blood sample was diluted with four times the amount of RPMI 1640 supplemented with 5\% FCS and antibiotics, at $\mathrm{pH} 7.4$ (Sigma-Aldrich, USA). The mixture was distributed in duplicate, in 96-sterile-well plate $(200 \mu \mathrm{l}$ per well). Twelve in vitro experimental variants were tested for each individual animal, namely (1) untreated control culture, (10) phytohaemagglutinin-M (PHA) $\left(1 \mu\right.$ per well), (3) thymus extract, (4) $70^{\circ}$ alcohol and (5-9) alcoholic vegetal extracts of Calendula officinalis, Arnica montana, Symphytum officinale, Echinacea purpurea and Echinaceea angustifolia, Balsam of Peru (11), bovine tuberculin (12) and Seleretard (1.5 $\mu \mathrm{l} /$ well).

The aliquots for all additions were established when using the same technique during preliminary studies as being the most effective in vitro. Subsequent to an incubation of $48 \mathrm{~h}$ at $37.5^{\circ} \mathrm{C}$ in a $5 \% \mathrm{CO}_{2}$ atmosphere, glucose consumption was evaluated. ${ }^{11,12}$ For this, $12.5 \mu \mathrm{l}$ of the cultural supernatant were transferred to $0.5 \mathrm{ml}$ of orto-toluidine reagent, boiled for $8 \mathrm{~min}$, cooled suddenly in cold water and read in a spectrophotometer at $610 \mathrm{~nm}$ wavelength (SUMAL PE2, Karl Zeiss, Jena, Germany), using the reagent as a blank. The stimulation/ inhibition index $(\mathrm{S} / \mathrm{I})$ was calculated as: $\mathrm{S} / \mathrm{I} \%=[(\mathrm{IG}-\mathrm{GR})$ $/ \mathrm{IG}]^{\prime} 100$, where $\mathrm{S} / \mathrm{I}=$ blast transformation index, $\mathrm{IG}=$ the initial glucose concentration in the culture medium and $\mathrm{GR}=$ glucose residue in the sample after incubation.

\section{Statistical analyses}

Average values and standard error were calculated by use of Excel program. Student's t test was applied to evaluate the statistical significance of the differences.

The black line indicates the average value of the group, showing that $62.5 \%$ of the animals have antibody values lower than the average (Figure 1).

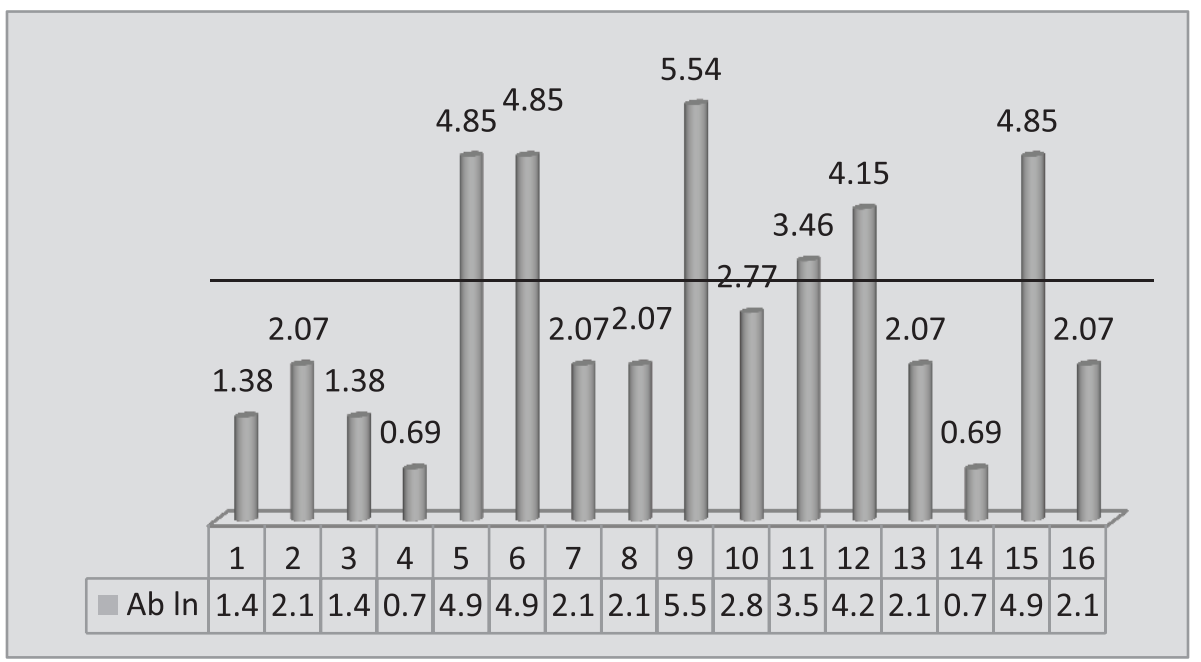

Figure 1: Individual variation of anti SRBC-antibody titres in primed foxes (In). 


\begin{tabular}{|c|c|c|}
\hline \multicolumn{2}{|c|}{ Table 1: Stimulation/inhibition indices in the leukocyte blast transformationtest $(\mathbf{x} \pm \mathbf{s})$} \\
\hline Variant & Unprimed group $(\mathbf{n = 1 4 )}$ & Primed group $(\mathbf{n}=16)$ \\
\hline Control & $17.49 \pm 22.9$ & $66.8 \pm 9.88$ \\
\hline PHA M & $-12.61 \pm 10.37$ & $6.67 \pm 29.0$ \\
\hline Thymus extract & $0.05 \pm 3.55$ & $28.37 \pm 23.64$ \\
\hline Alcohol & $-10.92 \pm 32.0$ & $27.63 \pm 23.46$ \\
\hline Calendula officinalis & $-14.71 \pm 10.73$ & $12.92 \pm 21.00$ \\
\hline Arnica montana & $-100.01 \pm 69.55$ & $-101.01 \pm 58.70$ \\
\hline Symphytum officinale & $-13.58 \pm 28.05$ & $-14.88 \pm 19.74$ \\
\hline Echinaceea purpureea & $-78.64 \pm 35.53$ & $-71.10 \pm 49.48$ \\
\hline Echinaceea angustifolia & $20.13 \pm 13.10$ & $20.28 \pm 21.27$ \\
\hline Balsam of Peru & $-140.89 \pm 121.77$ & $-162.84 \pm 58.44$ \\
\hline Bovine tuberculine & $35.41 \pm 19.76$ & $51.43 \pm 25.28$ \\
\hline Seleretard (barium selenite) & $37.0 \pm 15.79$ & $29.38 \pm 24.20$ \\
\hline
\end{tabular}

\section{RESULTS \& DISCUSSION}

The main mechanisms of defense against microbes involve antibodies directly blocking the pathogenic agent before it takes action against the host, promoting phagocytosis by micro- and macrophages and also involving Tlymphocytes in the process. These mechanisms could be induced by microbial challenge by either natural infection or vaccination, as part of disease prevention measures. Effects of antigen priming in Silver foxes were less studies. ${ }^{2-7-9}$

Farmed animals of wild origin are even more exposed to stress factors that interfere with their immune responses leaving them exposed to disease. All factors connected to farming lead to stress and subsequent failure of immunization.

Extracts from numerous plants were tested for their therapeutic and disease preventing potential. Plant extracts, as products with increased bioavailability and little or no side effects, could provide maximal stimulating and adjuvant activity; ${ }^{3}$ thus, vegetal extracts that could enhance the immune response to vaccines or during therapy are of utmost importance. ${ }^{15}$ Economic aspects on investments in vegetal adjuvants and drugs should be evaluated based on their therapeutic and immune active potential. . $^{4-10}$

The results of the Student t-test indicated significance of the differences between primed and unprimed groups for the blast transformation test at different levels ( $\mathrm{p}<0.001-\mathrm{p}<0.010$ ), only for the control, thymus extract and $C$. officinalis extract. The test also indicated that the effect of the extracts are strongly dependant on the plant genus, but also differ from species to species (Table1) Calendula officinalis and Echinaceea angustifolia leading to positive results.

The antibody synthesis induced by antigen priming did not lead to expected results. The antibody levels were highly variable, standing for the individual differences in the immune reactivity but also for the different perception of stress by these animals. In order to better protect these animals, antigen priming combined with plant extracts that positively acted in vitro could be of use. Necessarily, individualized protocols are required. The increased concentration of anti-SRBC antibodies also led to a non-significantly increased complexation $(109 \pm 4 \mathrm{U}$ and $41 \pm 1 \mathrm{U}, \mathrm{t}=1.709)$ in primed versus unprimed foxes.

\section{CONCLUSION}

SRBC exerted a positive effect on both humoral and cell-mediated immune responses in Silver foxes, supporting the enhancement of antimicrobial defense by booster vaccination and immune stimulating therapy, represented by vegetal extracts selected by host and plant species.

\section{ACKNOWLEDGEMENT}

This research was supported by grant PNII PNCD 61/2012 of MEN Romania.

\section{CONFLICT OF INTEREST}

The authors certify that they have no affiliations with or involvement in any organization or entity with any financial interest, or non-financial interest in the subject matter or materials discussed in this manuscript. 


\section{REFERENCES}

1. Badescu M, Badulescu O, Badescu L, Ciocoiu M. Effects of Sambucus nigra and Aronia melanocarpa extracts on immune system disorders within diabetes mellitus. Pharm Biol. 2015;53(4):533-9.

2. Black JG, Lawson KF. Sylvatic rabies studies in the silver fox (Vulpes vulpes). Susceptibility and immune response. Can J Comp Med. 1970;34(4):309-11.

3. Hilton LS, Bean AG, Lowenthal JW. The emerging role of avian cytokines as immuno-therapeutics and vaccine adjuvants. Vet Immunol Immunopathol. 2002;85(3-4):119-28

4. Jiang $\mathrm{ZH}$, Koganty RR. Synthetic vaccines: the role of adjuvants in immune targeting. Curr Med Chem. 2003;10(15):1423-39.

5. Kaufman PB, Cseke LJ, Warber S, Duke JA, Brielmann HL. Natural Products from Plants. CRC Press, Boca Raton, FL, 1999

6. Khokhlova IS, Spinu M, Krasnov BR, Degen AA. Immune response to fleas in a wild desert rodent: effect of parasite species, parasite burden, sex of host and host parasitological experience. The $\mathrm{J}$ of Experim Biol. 2004;207(16):2725-33

7. Nastoiu I. Fauna de interes medical, Bucharest, Editura All, 1994

8. Pádua TA, de Abreu BS, Costa TE, Nakamura MJ, Valente LMD, Siani AC, et al. Anti-inflammatory effects of methyl ursolate obtained from a chemically derived crude extract of apple peels: potential use in rheumatoid arthritis. Arch Pharm Res. 2014; 37(11):1487-95.
9. Rikula U, Pänkälä L, Jalkanen L, Sihvonen L. Distemper vaccination of farmed fur animals in Finland. Prev Vet Med. 2001;49(1-2):125-33.

10. Spînu Marina, Niculae Mihaela, Paştiu Anamaria loana, Şandru Carmen Dana, Pall Emoke, Vasiu A. Vegetal extracts influence in vitro on the cellmediated immunity in carnivores depending on health status, target species and plant taxonomy, Ind. Crops and Products. 2016;88:44-7.

11. Spînu M, Brudaşcă GHF, Şandru $C D$, Niculae $M$, Rindt K. Influences on the Cell-Mediated Response Following Hippophae rhamnoides Treatment, Analele SNBC Vol. XIII, CAP. IV - Imunologie. 2008;255-260

12. Spînu $M$, Brudaşcă GhF, Şandru $C D$, Niculae $M$, Ştefan $R$, Karolyi S. The immunological activity of non-conventional adjuvants, Clujul Medical Veterinar, 2008;(13-14):16-24.

13. Swain SS, Paidesetty SK, Padhy RN, Singh PK. Computational approach for locating effective cyanobacterial compounds against Mycobacterium tuberculosis, Indian Journal of Pharmaceutical Education and Research. 2017;51(2):302-11

14. Valcheva-Kuzmanova SV, Belcheva A. Current knowledge of Aronia melanocarpa as a medicinal plant. Folia Med (Plovdiv). 2006;48(2):11-7.

15. Vogel F.R. Improving vaccine performance with adjuvants; Clinical Infectious Diseases. 2000;30(suppl 3);266-70.

16. OIE. World Organization for Animal Health (2005) Manual of Diagnostic Tests and Vaccines for Terrestrial Animals. Available: http://web.oie.int/fr/normes/ mmanual /A_00037.htm. Accessed 12 February 2014.

\section{PICTORIAL ABSTRACT}

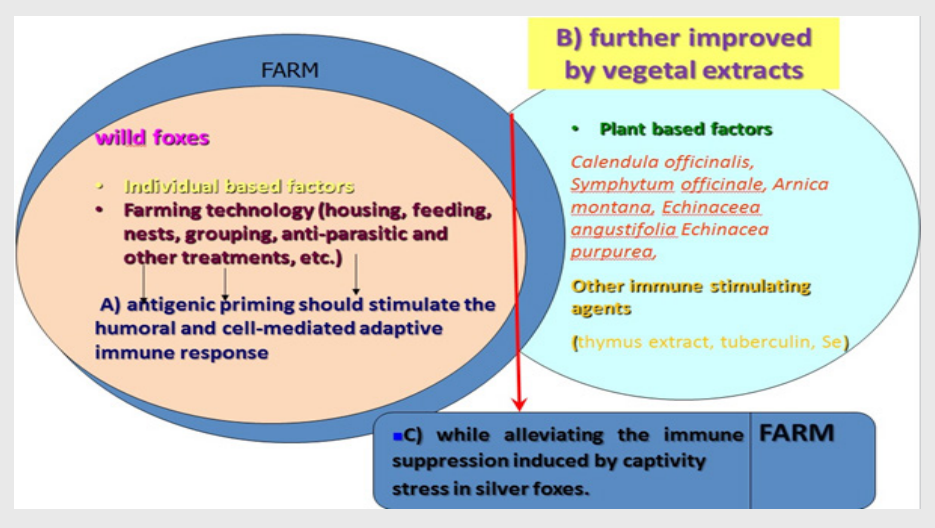

\section{SUMMARY}

- This study investigated the influence of several ethanolic vegetal extracts and $5 \%$ SRBC priming on in vitro cell-mediated reactivity, circulating immune complexes and antibody levels in farmed foxes ( $n=16$ primed and $n=14$ unprimed).

- The in vitro leukocytes blast transformation test was carried out using blood samples from foxes, while for antibody titers and circulating immune complexes sera were used.

- The effect of the vegetal extracts were strongly dependant on the plant genus, but also differed from species to species. Calendula officinalis (12.92 $\pm 21.00 \%)$ and Echinaceea angustifolia $(20.28 \pm 21.27 \%)$ lead to stimulation of the cellular immunity in the SRBC-primed group.

- The antibody levels were highly variable (from In 0.69 to In 5.54), standing for the individual differences in the immune reactivity in these animals.

- SRBC and vegetal extracts, selected by plant species, exerted a positive effect on both humoral and cell-mediated immune responses in Silver foxes, supporting the enhancement of antimicrobial defence by booster vaccination and immune stimulating therapy. 


\section{About Authors}
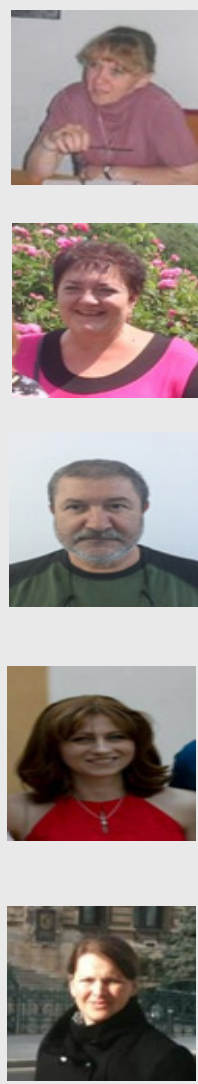

Emoke Pall, DVM, PhD: currently researcher with 11 years of experience, at the USAMV, Faculty of Veterinary Medicine, Cluj-Napoca, Romania. Areas of interest: infectious diseases, cell cultures, stem cells, microbiology/PCR (over 170 national and international publications).

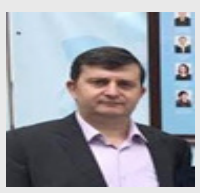

Aurel Vasiu, DVM, PhD: currently Lecturer in Infectious diseases, at the USAMV, Faculty of Veterinary Medicine, Cluj-Napoca, Romania. Areas of interest: infectious diseases, farmed animal pathology, microbiology (over 100 national and international publications).

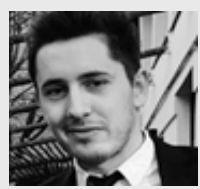

Constantin Cerbu, DVM, PhD student: currently Assistant with 3 years of teaching experience in Infectious diseases, at the USAMV, Faculty of Veterinary Medicine, Cluj-Napoca, Romania. Areas of interest: infectious diseases, microbiology of the environment, farm animal/fish pathology.

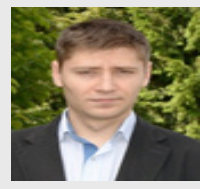

Radu Giupana, DVM: currently PhD student at the USAMV, Faculty of Veterinary Medicine, Cluj-Napoca, Romania. Areas of interest: infectious diseases, microbiology, bovine pathology, alternative therapies.

Cite this article: Spinu M, Niculae M, Pall E, Brudasca F, Vasiu A, Cerbu C, Giupana R, Sandru CD. The Potential Role of Antigen Priming In Increasing the Overall Antimicrobial Resistance in Captive Silver Foxes. Indian J of Pharmaceutical Education and Research. 2017;51(3)Suppl:S313-17. 